\title{
Duality of bounded and scattering wave systems with local symmetries
}

\author{
I. Kiorpelidis, ${ }^{1}$ F. K. Diakonos, ${ }^{1}$ G. Theocharis, ${ }^{2}$ V. Pagneux, ${ }^{2}$ O. Richoux,${ }^{2}$ P. Schmelcher,${ }^{3,4}$ and P. A. Kalozoumis ${ }^{1,2,5}$ \\ ${ }^{1}$ Department of Physics, University of Athens, 15771 Athens, Greece \\ ${ }^{2}$ LUNAM Université, LAUM, Université du Maine, CNRS, UMR No. 6613, Avenue O. Messiaen, 72085 Le Mans, France \\ ${ }^{3}$ Zentrum für Optische Quantentechnologien, Universität Hamburg, Luruper Chaussee 149, 22761 Hamburg, Germany \\ ${ }^{4}$ Hamburg Centre for Ultrafast Imaging, Universität Hamburg, Luruper Chaussee 149, 22761 Hamburg, Germany \\ ${ }^{5}$ Hellenic American University, 436 Amherst Street, Nashua, New Hampshire 03063, USA
}

(Received 24 September 2018; published 16 January 2019)

\begin{abstract}
We investigate the spectral properties of a class of hard-wall bounded systems, described by potentials exhibiting domainwise different local symmetries. Tuning the distance of the domains with locally symmetric potential from the hard-wall boundaries leads to extrema of the eigenenergies. The underlying wave function becomes then an eigenstate of the local symmetry transform in each of the domains of local symmetry. These extrema accumulate towards eigenenergies which do not depend on the position of the potentials inside the walls. They correspond to perfect transmission resonances of the associated scattering setup, obtained by removing the hard walls. We argue that this property characterizes the duality between scattering and bounded systems in the presence of local symmetries. Our findings are illustrated through a numerical example with a potential consisting of two domains of local symmetry, each comprised of Dirac $\delta$ barriers.
\end{abstract}

DOI: 10.1103/PhysRevA.99.012117

\section{INTRODUCTION}

The existence of symmetries provides numerous advantages to the study of a physical system, thereby yielding significant fundamental and phenomenological insights. The usual practice for most of the studied systems is to assume a global symmetry, i.e., the symmetry holds for the complete system under consideration. In this case, important properties, such as the band structure in periodic settings [1] or the classification into even and odd eigenstates for systems with global reflection (inversion) symmetry [2], can be extracted. However, due to the finite size of a realistic system as well as to the inevitable existence of defects, a globally valid symmetry constitutes an idealized scenario in nature. On the other hand, exact or approximate symmetries which exist in restricted spatial subdomains of a larger system are frequently met. Such spatially localized symmetries can be intrinsic in complex physical systems such as quasicrystals [3-5], partially disordered matter [6], large molecules [7,8], and biological materials [9].

Furthermore, contemporary technology requires structures with specialized properties which are not always possible to achieve in the presence of generic characteristics such as a global symmetry or total disorder. In such cases, local symmetries can be present by design, providing tailored properties and enhanced control in photonic multilayers [10-12], semiconductor superlattices [13], magnonic systems [14], and acoustic [15-17] and phononic [18-20] structures. With the term local symmetries (LSs) we refer to symmetries which are valid in spatial subdomains of the complete (embedding) space; one possible way is to consider them as remnants of a broken global symmetry.

The foundations of local symmetries and their impact in a variety of scattering systems have been investigated in a se- quence of recent works. A rigorous mathematical framework for the description of symmetry breaking leading to local symmetries has been developed in Refs. [21-23], where nonlocal invariant currents have been identified as remnants of broken global symmetries. In Ref. [24] it was shown that the longrange order and complexity of lattice potentials generated by well-known binary aperiodic one-dimensional sequences can be encapsulated within their local symmetry structure, while in Ref. [25] the case of driven lattices was discussed. The scattering properties of quantum and photonic aperiodic structures were discussed in Refs. [26,27], answering the puzzling question about the existence of perfect transmission resonances in aperiodic systems and also providing a classification scheme with respect to their kind. These theoretical findings were experimentally verified in Ref. [28] in the framework of acoustic waveguides. Apart from continuous scattering systems, the impact of local symmetries has been also investigated in the framework of discrete systems [29]. In this context, the local symmetry partitioning revealed possibilities for the design of flat bands and compact localized states [30]. Very recently, it was shown that the existence of local symmetries plays a crucial role in the real-space control of edge states in aperiodic chains [31] as well as in the wave delocalization and transport between disorder and quasiperiodicity [32]. Thus, the concept of local symmetries, even being a recent one, has already led to a rich phenomenology and revealed properties of fundamental importance.

Even though local symmetries in continuous scattering and discrete systems have been extensively investigated in the aforementioned works, their consequences and effects in continuous bounded systems remain unexplored. The link between bounded and unbounded systems is a long-standing subject of study in quantum mechanics and in the more general context of wave physics [33]. Several methods have been 
employed to describe how a bounded system is connected to its open counterpart [34], since spectral properties usually can be measured when the system is coupled to an environment, leading, for instance, to the inside (bound states) -outside (scattering) duality [35-37]. Another way to view and study the bound-scattering duality is through the notion of quasistates, namely, scattering resonances which become actual bound states when the system closes [38,39]. Nonetheless, here this link is studied under the prism of local symmetries.

As a step in this direction we explore in this work the properties of one-dimensional bounded systems with two locally symmetric potential barriers, focusing on the case of local reflection symmetries. We define as the setup the two locally symmetric potential barriers along with the distance which separates them, while the term system is used to describe the entire potential landscape consisting of both the setup and the bounding hard walls. Tuning the distance of the setup from the left hard wall, we prove the existence of spectral extrema where the mirror symmetry of the wave function is restored inside each reflection symmetric potential barrier. We also establish a link between the spectral properties of a generic bounded system with two domains of local symmetry and the properties of the respective scattering system. In particular, we find that certain eigenenergies of the bounded system correspond to the energies where perfect transmission resonances (PTRs) manifest in the transmittance of its scattering counterpart and we prove that these eigenenergies are unaffected by the position change of the setup inside the walls. These theoretical findings are numerically verified for a system with two domains of local reflection symmetry comprised of Dirac $\delta$ barriers.

The paper is organized as follows. In Sec. II we summarize the key ingredients and present some basic results of scattering theory in systems with local symmetries. Also we introduce the setups which we will employ, both in the scattering and in the bounded context. In Sec. III we focus on the properties of a bounded system with local symmetries comprised of Dirac $\delta$ barriers and discuss the relevant properties. We also discuss the connection between certain bounded states and PTRs. In Sec. IV we generalize rigorously our results for a generic system with two domains of local symmetry of arbitrary potential shape. Our results are summarized in Sec. V.

\section{OVERVIEW OF SCATTERING IN SYSTEMS WITH LOCAL SYMMETRIES}

\section{A. Perfect transmission resonances}

Scattering potentials possessing a global mirror symmetry and the possibility of the occurrence of PTRs have been directly linked to each other in several studies [40,41]. On the other hand, the lack of such a symmetry usually leads to a nonvanishing reflection of an incoming scattering wave. However, the existence of PTRs in aperiodic [12] structures possessing no global mirror symmetry has been reported. Recently, we established [27] a classification of the possible PTRs which occur in nonglobally symmetric systems. In particular, for a system with local symmetries the PTRs can be classified according to the symmetry of the wave-function modulus
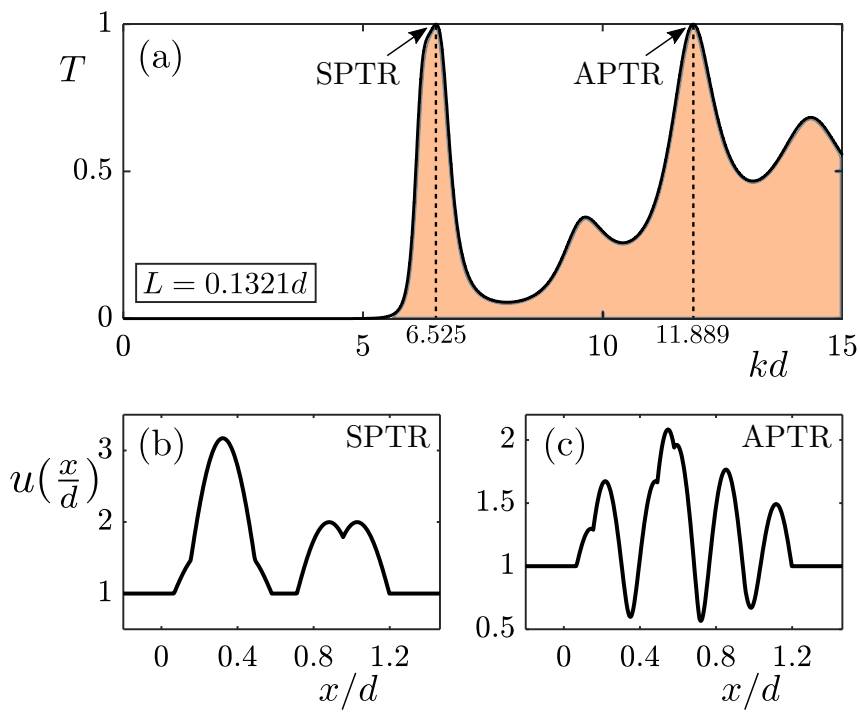

FIG. 1. (a) Transmittance setup. The two peaks correspond to a SPTR and an APTR, respectively. Also shown is the magnitude of the wave function at the wave numbers of the (b) SPTR and (c) APTR shown in (a). Here $D / d=1.2644$ (and $L / d=0.1321$ ).

$u(x)=|\psi(x)|$. If $u(x)$ is reflection symmetric within the domains of local reflection symmetry then it is called a symmetric PTR (SPTR), whereas if $u(x)$ does not obey this local symmetry it is called asymmetric PTR (APTR). In the SPTR case each domain of local symmetry is individually transparent. For APTRs the system is transparent only as a whole.

Figure 1(a) shows the transmittance of the setup shown in Fig. 2(a). The two peaks correspond to a SPTR and an APTR, as their wave-function moduli indicate in Figs. 1(b) and 1(c), respectively. Note here that the PTRs shown in Fig. 1(a) do not occur by chance. The setup is designed according to prescription based on local symmetries and the parameters (indicated in Fig. 2) are suitably tuned in order to emerge at the specific frequencies. Also, a different kind of tuning is required for an APTR and a SPTR, respectively. This design technique and a thorough investigation of the scattering properties of the system which corresponds to the transmittance shown in Fig. 1(a) can be found in Ref. [28].

\section{B. Symmetry-induced invariant currents}

Another important finding of our theoretical framework on local symmetries is the existence of symmetry-induced currents which are spatially invariant in domains where a certain symmetry, i.e., reflection, translation, or $\mathcal{P} \mathcal{T}$ symmetry, is present. Employing a generic wave mechanical framework, we consider a generalized Helmholtz equation $\psi^{\prime \prime}(x)+$ $U(x) \psi(x)=0$, where $U(x)$ is the generalized potential. In this framework, it is possible to treat in a unified way different wave mechanical systems of, e.g., photonic, acoustic, and quantum mechanical origin. Assuming that the potential $U(x)$ obeys a symmetry transformation $U(x)=U[F(x)]$ within a domain $\mathcal{D} \subseteq \mathbb{R}(\mathcal{D}=\mathbb{R}$ corresponds to a global symmetry), it 

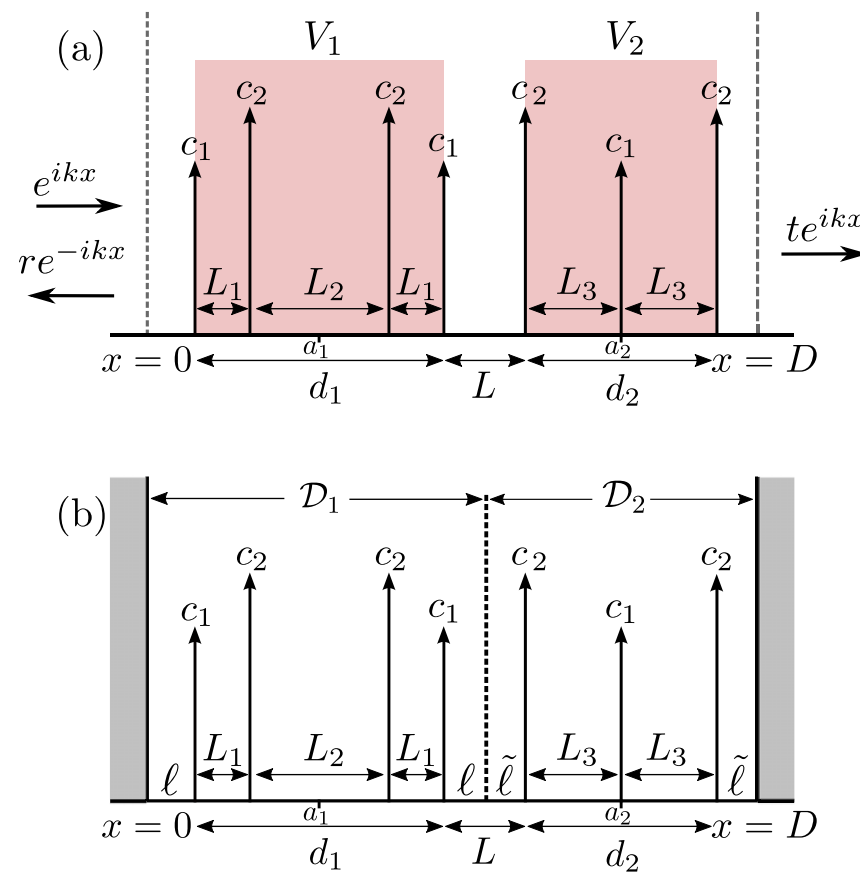

FIG. 2. (a) Scattering and (b) bounded systems with local symmetries. Each potential subpart $V_{1}$ and $V_{2}$ is reflection symmetric. The distances between the $\delta$ functions are equal to $L_{1} / d=$ $0.0884, L_{2} / d=0.3372, L / d=0.1321$, and $L_{3} / d=0.2431$ and the strengths of the $\delta$ barriers are equal to $c_{1}=6.2492 / d$ and $c_{2}=$ $9.7766 / d$.

can be shown that a spatially invariant nonlocal current exists,

$$
\mathcal{Q}=\frac{1}{2 i}\left[\sigma \psi(x) \psi^{\prime}(\tilde{x})-\psi(\tilde{x}) \psi^{\prime}(x)\right]=\mathrm{const} \forall x, \quad \tilde{x} \in \mathcal{D} .
$$

This quantity plays a fundamental twofold role. It provides the tool to systematically describe the breaking of discrete symmetries, while it also generalizes the Bloch and parity theorems for systems with broken translation and reflection symmetry, respectively [21]. The quantity $\mathcal{Q}$ is of central importance for this study. Note that, in bounded systems, since the wave function is real, only the invariant current $\mathcal{Q}$ exists. On the other hand, in scattering systems where the wave function $\psi(x)$ is complex, an additional invariant quantity

$$
\tilde{\mathcal{Q}}=\frac{1}{2 i}\left[\sigma \psi^{*}(x) \psi^{\prime}(\tilde{x})-\psi(\tilde{x}) \psi^{\prime *}(x)\right]=\mathrm{const} \forall x, \quad \tilde{x} \in \mathcal{D}
$$

emerges.

\section{Description of the setup}

Let us now describe the setup which we will use throughout this work. Figure 2(a) illustrates a scattering system comprised of seven Dirac $\delta$ barriers, forming two reflection symmetric potential subparts denoted by $V_{1}$ and $V_{2}$ (colored areas). The lengths of $V_{1}$ and $V_{2}$ are $d_{1}$ and $d_{2}$, respectively, while their separating distance is $L$. Moreover, $a_{1}$ and $a_{2}$ stand for the positions of the reflection centers of each subpart. The parameters $c_{i}, i=1,2$, represent the strength of the $\delta$ barriers and $r$ and $t$ are the reflection and transmission coefficients, respectively. A detailed study of the scattering properties of this system and their experimental verification can be found in Ref. [28]. Figure 2(b) shows the corresponding bounded system, where the aforementioned setup is delimited by hardwall boundaries. The general characteristics remain the same. However, the distance $\ell$ which determines the distance from the left wall plays a crucial role and in the following will serve as our tuning parameter. In order to preserve the local symmetries of the system for any value of $\ell$, namely, the domains $\mathcal{D}_{1}$ and $\mathcal{D}_{2}$ being always reflection symmetric, $L=$ $\ell+\tilde{\ell}$ should hold [see Fig. 2(b)].

At this point we would like to emphasize the fact that the duality between bounded and scattering systems, which is established in this work, is not restricted to systems comprised of $\delta$ barriers. In fact, it is general and valid for systems with two domains of local reflection symmetry with potential barriers of arbitrary shape, as it will be proven in Sec. IV.

\section{LOCAL SYMMETRIES IN BOUNDED SYSTEMS}

Several connections between bounded and scattering systems can be investigated. In this work we focus on bounded systems and how they can be linked to their scattering counterparts from the perspective of PTRs and local symmetries. To this end, we consider the bounded version of our system as shown in Fig. 2(b). We remind the reader that for the lengths $\ell$ and $\tilde{\ell}, L=\ell+\tilde{\ell}$ holds. With this choice, and employing $\ell$ as our tuning parameter with $\ell \in[0, L]$, we ensure that for any value of $\ell$ the system is always decomposable into two locally symmetric domains $\mathcal{D}_{1}$ and $\mathcal{D}_{2}$. Keeping $L$ fixed and varying $\ell$, we expect that the spectrum of the allowed wave numbers will change continuously. During this variation we will examine the spectral properties which emerge due to the local symmetries. In order to render all quantities dimensionless, we divide lengths and multiple wave numbers by the distance $d=d_{1}+d_{2}$, which is the sum of the width of the two reflection symmetric potential barriers. We choose this distance since it remains unaffected by the shifts of the setup inside the boundaries.

Figure 3(a) shows the first six states of the bounded system [see Fig. 2(b)] for $L / d=0.1321$ and how these change as $\ell$ varies within the range $[0, L]$. The small figures on the right provide a close-up of the selected curves for better resolution. A striking characteristic is the perfectly flat black solid line which indicates that the wave number $\kappa$ remains unaffected by the position of the setup inside the box. This is an important observation because eigenenergies with this property emerge in the respective scattering counterpart as PTRs, offering the ground for establishing a duality between open and closed systems. Figure 3(b) shows the transmittance of the corresponding scattering system. The dashed line indicates an APTR peak at $k=11.889 / d$. In Fig. 3(c) we focus on the fourth state of Fig. 3(a) and examine its behavior for several $L$ values in the vicinity of $L / d=0.1321$. For $L / d<0.1321$ each $\kappa(\ell / d)$ curve exhibits a minimum. Exactly at $L / d=0.1321$ the wave number $\kappa=11.889 / d$ becomes invariant with respect to the position of the setup within the walls and this manifests through the flat solid line. Remarkably, this $\kappa$ value is identical to the $k$ value of the APTR in the transmittance of Fig. 3(b). For $L / d>0.1321$ 

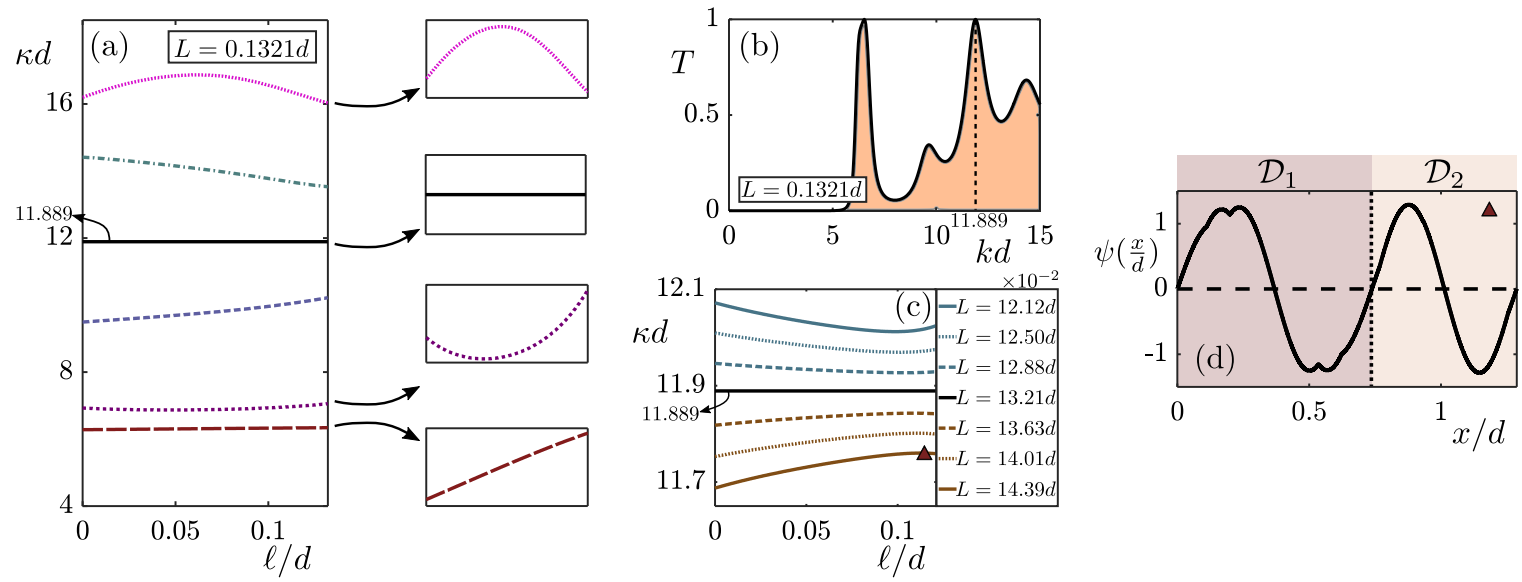

FIG. 3. (a) Spectrum showing the first six states of the bounded system shown in Fig. 2(b) for $L / d=0.1321$. As the tuning parameter $\ell$ varies in the range $[0, L]$ the wave number $\kappa$ varies continuously. The insets zoom into the curves to provide better resolution. (b) Transmittance for the corresponding open system. The dashed line at $k=11.889 / d$ indicates an APTR. (c) Fourth state of the spectrum of the bounded system for different $L$ values. For $L / d=0.1321$ a duality between the open and closed systems occurs connecting the PTR wave number $k=11.889 / d$ and the invariant bounded state $\kappa=11.889 / d$. (d) Wave function which follows the local symmetries of the setup at the maximum indicated with the triangle.

the $\kappa(\ell / d)$ curves exhibit maxima. All extrema approach the flat line of $L / d=0.1321$.

It is obvious that for each $L$ value, both the spectrum of the bounded system and the transmittance of the scattering system will change. Nevertheless, the aforementioned correspondences can be identified. In Fig. 4 the respective properties of the system for $L / d=0.3025$ are discussed. Figure 4(a) shows five states of the spectrum and how these change as $\ell$ varies within the range $[0, L]$. The eigenstate which is invariant under $\ell$ shifts corresponds to the SPTR shown in the transmittance of Fig. 4(b). In Fig. 4(c) the dependence of the second state as $L$ changes is shown. The pattern is the same as in the previous example. For $L / d=0.3025$ the wave number $\kappa=6.525 / d$ remains constant as the $\ell$ changes. For this $L$ value the transmittance exhibits a SPTR peak at the same wave number $k=6.525 / d$.
For $L / d \neq 0.3025, \kappa(\ell / d)$ possess extrema, which saturate to the flat line.

Another very interesting property which is observed here relates the extrema of the $\kappa(\ell / d)$ curves with the local symmetries of the setup and the form of the wave function. In particular, at every extremum of the $\kappa(\ell / d)$ curves, the wave function becomes an eigenstate of the local reflection symmetry transform and follows the local symmetries of the setup. Figures 3(c) and 3(d) illustrate this case. In particular, Fig. 3(d) shows the wave function at the maximum of the curve for $L / d=0.1439$ (the correspondence is indicated by the $\Delta$ ). It is clear that the wave function is (locally) parity definite within the local symmetry domains $\mathcal{D}_{1}$ and $\mathcal{D}_{2}$ of the setup. The same holds for the system in Fig. 4. The wave function in Fig. 4(d) corresponds to the minimum of the $\kappa(\ell / d)$ curve for $L / d=0.2840$ (see the $\nabla$ ) and is locally
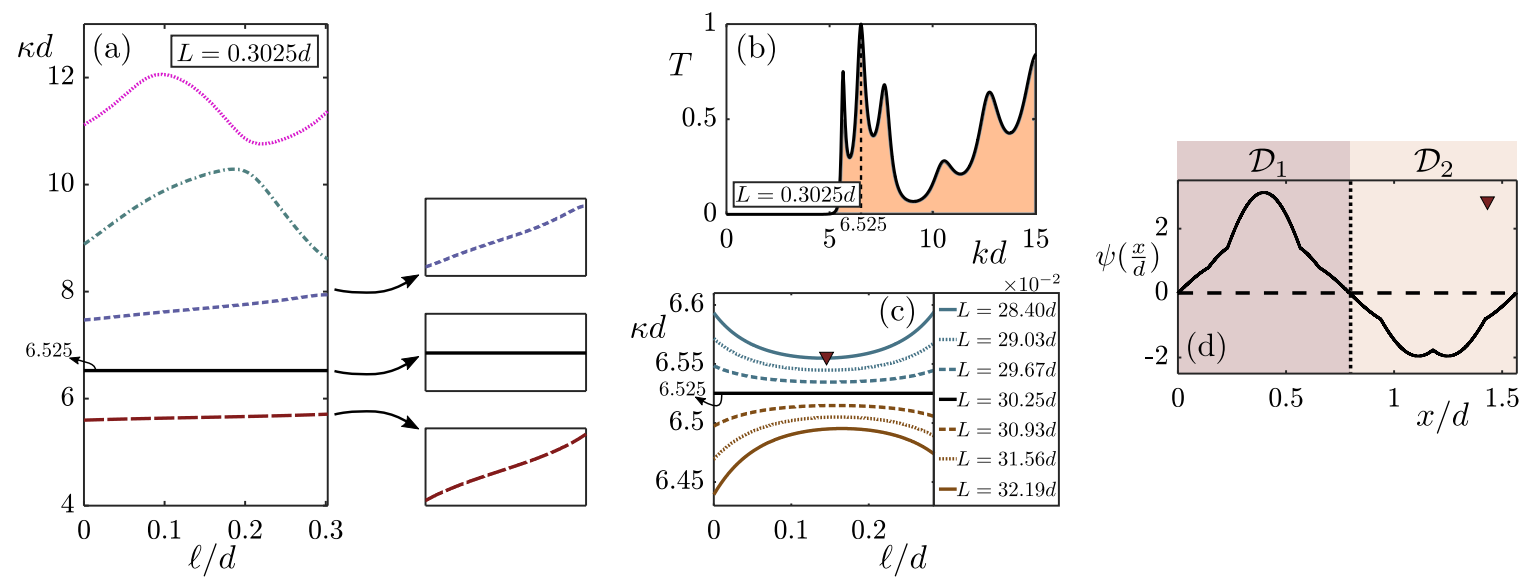

FIG. 4. (a) Spectrum showing five states of the bounded system shown in Fig. 2(b), this time for $L / d=0.3025$. As $\ell$ varies in $[0, L]$ the wave number $\kappa$ varies continuously. (b) Transmittance for the corresponding open system. The dashed line at $k=6.525 / d$ indicates an SPTR. (c) Second state of the spectrum of the bounded system for different $L$ values. For $L / d=0.3025$ a duality between the open and closed systems occurs connecting the PTR wave number $k=6.525 / d$ and the invariant bounded state $\kappa=6.525 / d$. (d) Wave function which follows the local symmetries of the setup at the minimum indicated with the down triangle. 

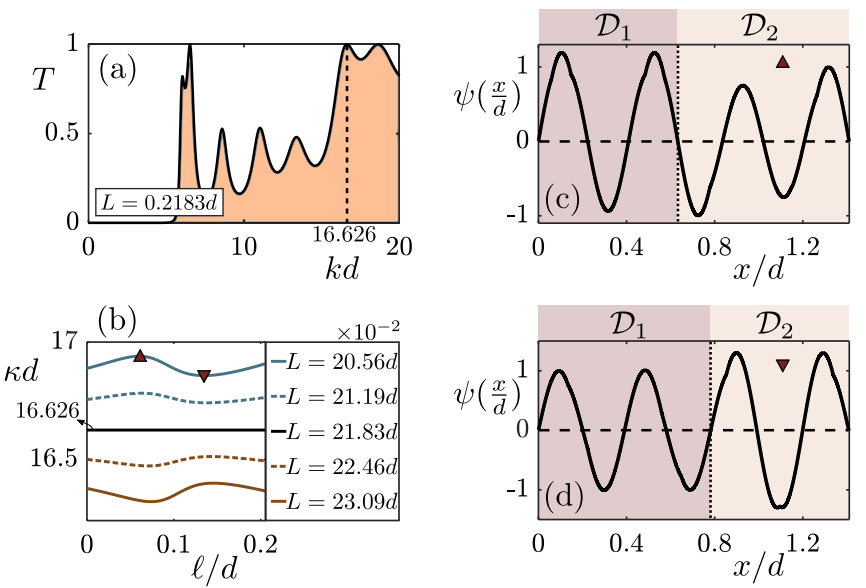

FIG. 5. (a) Transmittance of the setup shown in Fig. 2(a) for $L / d=0.2183$. The dashed line corresponds to a PTR which occurs for the specific $L / d=0.2183$ choice at $k=16.626 / d$. (b) Variation of the seventh state with $\ell / d$ of the spectrum of the respective bounded system for several $L$ choices around $L / d=0.2183$. Each $\kappa d$ vs $\ell / d$ curve exhibits one minimum and one maximum. For $L / d=0.2183$ the wave number $(\kappa=16.626 / d)$ is independent of $\ell$ with a value coinciding with the wave number $k$ of the APTR. Also shown are the wave functions at the (c) maximum and (d) minimum of the first curve (indicated by up and down triangles, respectively). Here the wave function becomes an eigenstate of the local symmetry transform.

parity definite following the symmetries of the $\mathcal{D}_{1}$ and $\mathcal{D}_{2}$. This correspondence between the $\kappa(\ell / d)$ extrema and the local symmetry properties of the wave function provides a systematic attempt to investigate the manifestation of local symmetries in bounded systems.

In fact, there are $\kappa(\ell / d)$ curves which may possess more than one extrema, such as those shown in Fig. 5(b). These curves correspond to the seventh state of a bounded system for several $L$ values around $L / d=0.2183$. The flat line occurs for this $L$ at $\kappa=16.626 / d$ and again benchmarks the appearance of an APTR in the corresponding scattering setup, as indicated in Fig. 5(a), a finding which supports the duality between open and closed systems at the PTR wave numbers. Figures 5(c) and 5(d) illustrate the wave function at the two extrema of the first curve (marked with the up and down triangles). In both cases, it becomes parity definite within the two domains of local symmetry $\mathcal{D}_{1}$ and $\mathcal{D}_{2}$. Note that the fifth state in Fig. 4(a) also possesses two extrema. However, we showed the case of a different setup in order to stress further the correspondence between PTRs and translationally invariant bound states.

Note that this bound-scattering duality and the manifestation of local symmetries on the extrema are not system specific. Our conclusions will be rigorously proven and generalized for systems with two locally symmetric potential domains of arbitrary shape in the following section. To conclude this section we summarize our key findings in the following, which hold for the general case. Consider a bounded system which consists of two domains of local symmetry $\mathcal{D}_{1}$ and $\mathcal{D}_{2}$, each with a reflection symmetric potential $V_{1}$ and $V_{2}$ of arbitrary shape and finite support. Between this system

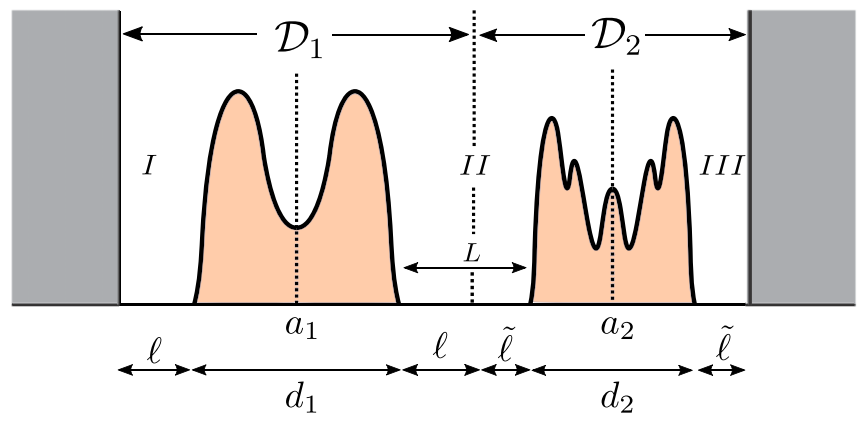

FIG. 6. Schematic of a bounded system with hard-wall boundary conditions containing two domains of local symmetry $\mathcal{D}_{1}$ and $\mathcal{D}_{2}$. In regions I-III the potential vanishes.

and its scattering counterpart the following duality holds. (i) Starting from a bounded system, if a bound state with wave number $\kappa$ is invariant with respect to translations of the setup inside the cavity, then it corresponds to a PTR (asymmetric or symmetric) in the corresponding scattering system with incoming wave number $k=\kappa$. (ii) Starting from a scattering system, the existence of an APTR in a scattering system at $k$ is equivalent to a bound state with wave number $\kappa=k$ which is invariant under translations of the (same) setup inside the cavity. The reason that we discriminate SPTRs from APTRs is because the wave number $k$ of an APTR will always emerge as an eigenstate with wave number $\kappa$ in the corresponding bounded system. This one-to-one correspondence between scattering and bounded systems exists because the APTR occurs for a specific distance $L$ between the two locally symmetric scatterers. For the SPTR, on the other hand, this one-to-one correspondence between scattering and bounded systems would not be possible because it appears in the transmittance for any distance $L$ between the locally symmetric scatterers [27]. In this case, all bounded systems' spectra for all different $L$ values would have an eigenstate at the same $\kappa$, which is not possible. Nevertheless, if the bounded system has an eigenstate at a $\kappa$ value which coincides with the $k$ wave number of an SPTR of its scattering counterpart, then the equivalence between the SPTR and the bound-state translation invariance is preserved (case shown in Fig. 4). (iii) All extrema which emerge in the $\kappa(\ell / d)$ curves correspond to states which are eigenstates of the local reflection symmetry transform and the wave function is parity definite inside $\mathcal{D}_{1}$ and $\mathcal{D}_{2}$.

\section{GENERALIZATION FOR AN ARBITRARY BOUNDED SYSTEM WITH TWO DOMAINS OF LOCAL SYMMETRY}

In this section we will generalize the results presented above for arbitrary bounded systems with two domains of local symmetry. In order to prove the above statement we employ the transfer matrix (TM) approach to connect the wave fields in the regions I, II, and III of the system, as shown in Fig. 6. Since I-III are potential-free regions, the wave function will be of the form

$$
\psi_{m}(x)=A_{m} e^{i \kappa x}+B_{m} e^{-i \kappa x}, \quad m=\mathrm{I}, \mathrm{II}, \mathrm{III} .
$$


The connection between $\psi_{\mathrm{I}}$ and $\psi_{\mathrm{II}}$ is provided by the TM, which reads, for a Hermitian system,

$$
M_{\mathcal{D}_{j}}=\left[\begin{array}{cc}
w_{j} & z_{j} \\
z_{j}^{*} & w_{j}^{*}
\end{array}\right], \quad j=1,2 .
$$

Here $j=1,2$ corresponds to the TMs of the LS symmetric subparts defined on $\mathcal{D}_{1}$ and $\mathcal{D}_{2}$, respectively. For the TM elements it holds that $w_{j}=1 / t_{j}$ and $z_{j}=r_{j}^{*} / t_{j}^{*}$, where $t_{j}$ and $r_{j}$ are the transmission and reflection amplitudes of the $j$ th potential unit, respectively.

Positioning the first wall at $x=0$, the wave function should be zero there, i.e., $\psi(0)=0$, leading to the condition $A_{\mathrm{I}}=-B_{\mathrm{I}}$ and subsequently to

$$
\frac{A_{\mathrm{II}}}{B_{\mathrm{II}}}=-\frac{z_{1 c} e^{-2 i \kappa a_{1}}+w_{1 c}^{*}}{z_{1 c}^{*} e^{2 i \kappa a_{1}}+w_{1 c}},
$$

connecting regions I and II. Note here that the index $c$ in the TM elements (referring to centered) corresponds to the TM for $\mathcal{D}_{1}$ centered at $x=0$. The shift to the actual position of $\mathcal{D}_{1}$ in our setup is realized by the phases in Eq. (5), with $a_{1}$ being the position of the reflection axis of $\mathcal{D}_{1}$.

In the same manner, taking the wave function to be zero at the second wall $\psi(D)=0$, we find the condition $B_{\mathrm{III}}=$ $-A_{\mathrm{III}} e^{2 i k D}$, which in turn leads to the relation

$$
\frac{A_{\mathrm{II}}}{B_{\mathrm{II}}}=\frac{w_{2 c}-z_{2 c} e^{-2 i \kappa a_{2}} e^{2 i \kappa D}}{z_{2 c}^{*} e^{2 i \kappa a_{2}}-w_{2 c}^{*} e^{2 i \kappa D}},
$$

connecting the plane-wave coefficients in regions II and III. Here $a_{1}$ and $a_{2}$ correspond to the mirror symmetry center of the two scatterers (see Fig. 6). In turn, Eqs. (5) and (6) yield

$$
\mathcal{G}=\frac{z_{1 c} e^{-2 i \kappa a_{1}}+w_{1 c}^{*}}{z_{1 c}^{*} e^{2 i \kappa a_{1}}+w_{1 c}}+\frac{w_{2 c}-z_{2 c} e^{-2 i \kappa a_{2}} e^{2 i \kappa D}}{z_{2 c}^{*} e^{2 i \kappa a_{2}}-w_{2 c}^{*} e^{2 i \kappa D}}=0,
$$

which involves only the wave number $\kappa$ and the characteristic parameters of the system which are included in the TM elements $w_{j c}$ and $z_{j c}(j=1,2)$. In order to facilitate the mathematical computations we consider only the numerator of Eq. (7), which we set as $\mathcal{F}$. Since we are interested in the behavior of $\kappa$ with respect to $\ell$ it is sufficient to calculate the total derivative of $\mathcal{F}$ with respect to $\ell$ and then calculate the derivative $d \kappa / d \ell$,

$$
\frac{d \mathcal{F}}{d \ell}=\frac{\partial \mathcal{F}}{\partial \ell}+\frac{\partial \mathcal{F}}{\partial \kappa} \frac{d \kappa}{d \ell}=0
$$

which leads to

$$
\frac{d \kappa}{d \ell}=-\frac{\frac{\partial \mathcal{F}}{\partial \ell}}{\frac{\partial \mathcal{F}}{\partial \kappa}}
$$

Therefore, the behavior of the wave number $\kappa$ with respect to $\ell$ can be investigated via the term $\partial \mathcal{F} / \partial \ell$. Note that in order to find $d \mathcal{F} / d \ell$ we have expressed $a_{1,2}$ with respect to $\ell, d_{1}, d_{2}$, and $L$, namely, $a_{1}=\ell+d_{1} / 2$ and $a_{2}=\ell+d_{1}+L+d_{2} / 2$. Then the latter is written as

$$
\begin{aligned}
\partial \mathcal{F} / \partial \ell= & 2 i \kappa\left[e^{i \kappa \mathcal{P}}\left(w_{1 c}^{*} z_{2 c}^{*}+z_{1 c}^{*} w_{2 c} e^{-i \kappa D}\right)\right. \\
& \left.+e^{-i \kappa \mathcal{P}}\left(w_{1 c} z_{2 c}+z_{1 c} w_{2 c}^{*} e^{i \kappa D}\right)\right],
\end{aligned}
$$

where $\mathcal{P}=2 \ell+d_{1}$. In the following we will show that this equation has a very instructive form regarding the emergence of local symmetries. To this end, we employ the existence of the symmetry-induced invariant current $\mathcal{Q}$ for reflection symmetry, as defined in Eq. (1). Given the plane-wave solution in the potential-free regions I-III [see Eq. (3)], we find that the form for $\mathcal{Q}_{1}$ and $\mathcal{Q}_{2}$ in the LS domains $\mathcal{D}_{1}$ and $\mathcal{D}_{2}$ is

$$
\begin{aligned}
& \mathcal{Q}_{1}=\kappa\left(A_{\mathrm{I}} A_{\mathrm{II}} e^{2 i \kappa a_{1}}+B_{\mathrm{I}} B_{\mathrm{II}} e^{-2 i \kappa a_{1}}\right), \\
& \mathcal{Q}_{2}=\kappa\left(A_{\mathrm{II}} A_{\mathrm{III}} e^{2 i \kappa a_{2}}+B_{\mathrm{II}} B_{\mathrm{III}} e^{-2 i \kappa a_{2}}\right),
\end{aligned}
$$

where $A_{j}$ and $B_{j}$ are the plane-wave coefficients in regions I-III and $\kappa$ is the wave number of the specific eigenstate. We stress here that $\mathcal{Q}$ can be calculated taking into account only the potential-free regions I-III, since, due to the symmetry, it is independent of the exact potential form (see Ref. [21]). Note also that Eqs. (11a) and (11b) have no dependence on $x$, signaling its spatial invariance. Focusing on the setup shown in Fig. 1 , we find that $\mathcal{Q}_{1}$ for the domain $\mathcal{D}_{1}$ reads

$$
\mathcal{Q}_{1}=k A_{\mathrm{I}} B_{\mathrm{II}}\left(\frac{A_{\mathrm{II}}}{B_{\mathrm{II}}} e^{2 i \kappa a_{1}}+e^{-2 i \kappa a_{1}}\right),
$$

where we have used the condition $B_{\mathrm{I}}=-A_{\mathrm{I}}$. The behavior of $\mathcal{Q}_{1}$ determines the LS properties of the wave function in the domain $\mathcal{D}_{1}$. In particular, if $\mathcal{Q}_{1}=0$ the wave function inside $\mathcal{D}_{1}$ will be parity definite. Substituting Eq. (5) into Eq. (12) and setting $\mathcal{Q}_{1}=0$, we find

$$
z_{1 c}=\frac{1}{2}\left(w_{1 c} e^{-i \kappa \mathcal{P}}-w_{1 c}^{*} e^{i \kappa \mathcal{P}}\right),
$$

where we have used the property $z_{1 c}=-z_{1 c}^{*}$, which holds for the TM of reflection symmetric potentials. Following the same procedure for the domain $\mathcal{D}_{2}$ and for $\mathcal{Q}_{2}=0$, we find

$$
z_{2 c}=\frac{1}{2}\left(w_{2 c} e^{i \kappa \mathcal{P}} e^{-i \kappa D}-w_{2 c}^{*} e^{-i \kappa \mathcal{P}} e^{\kappa k D}\right) .
$$

We stress here that when Eqs. (13) and (14) hold, then the wave function is parity definite inside $\mathcal{D}_{1}$ and $\mathcal{D}_{2}$, respectively.

The next step is to substitute Eqs. (13) and (14) into Eq. (10). This allows us to focus on the behavior of the quantity $\partial \mathcal{F} / \partial \ell$ when the wave function is parity definite in both domains $\mathcal{D}_{1}$ and $\mathcal{D}_{2}$. After some algebraic manipulation, we find that $\partial \mathcal{F} / \partial \ell=0$ and in turn that

$$
d \kappa / d \ell=0 \text {. }
$$

Therefore, the restoration of LS in the wave function (i.e., the field is parity definite inside $\mathcal{D}_{1}$ and $\mathcal{D}_{2}$, and $\mathcal{Q}_{1}=\mathcal{Q}_{2}=0$ ) in any structure comprised of two barriers of arbitrary shape obeying the corresponding local symmetry manifests as an extremum in the $\kappa$ vs $\ell$ curve.

The implications of Eq. (15) on bounded systems provide also certain interesting links to their corresponding scattering counterparts. The transition from the bounded system to the scattering one is achieved by removing the hard-wall boundaries, leaving the system otherwise unaffected. Then, on either side of the setup, the potential vanishes and the wave function can be described by plane waves. Here the asymptotic conditions are described by incoming and outgoing waves of the form $\psi_{\mathrm{I}}(x)=e^{i k x}+r e^{-i k x}$ and $\psi_{\mathrm{III}}(x)=$ $t e^{i k x}$, respectively. Note that $k$ is the continuous wave number of the scattering system, while $r$ and $t$ are the transmission amplitudes.

Then we can distinguish two cases of particular interest which render Eq. (10) [and consequently Eq. (15)] also equal 
to zero. These cases provide the connection between special spectral points of the bounded system and the wave numbers where PTRs occur in the transmittance of the respective scattering system. For clarity, we denote by $k_{s}$ and $k_{a}$ the wave numbers where the SPTR and APTR occur, respectively. Therefore, we have the following $\kappa$ values.

(i) $\kappa=k_{s}$. At this $\kappa$ value the corresponding scattering system exhibits a SPTR. In the case of a SPTR both potential parts are independently transparent (for a detailed analysis see Ref. [27]). The independence refers to the fact that their distance $L$ is irrelevant to their transparency. In terms of the TM formalism, this occurs when the antidiagonal terms are $z_{1 c}\left(k_{s}\right)=z_{2 c}\left(k_{s}\right)=0$. In this case $\left(\kappa=k_{s}\right)$ Eqs. (10) and (15) become zero independently of the $\ell$ value. Therefore, the eigenstate with $\kappa=k_{s}$ will be invariant under translations of the setup inside the cavity and the $\kappa$ vs $\ell / L$ curve will appear as a horizontal line. We recall here that for the scattering system the SPTR at $k_{s}$ will appear for any distance $L$ between the two scatterers. On the other hand, for the bounded system not all $L$ values will yield an eigenstate at $\kappa=k_{s}$. If, however, an eigenstate with $\kappa=k_{s}$ exists in the spectrum, then it will have the aforementioned translation invariance property.

(ii) $\kappa=k_{a}$. At this $\kappa$ value the corresponding scattering system exhibits an APTR. In the case of an APTR the complete setup, i.e., the combination of the two subparts, is transparent. Then the off-diagonal terms of the total TM, which is the product $M_{\mathcal{D}_{1}} \times M_{\mathcal{D}_{2}}$ of the two individual TMs, is given by $z_{\text {tot }}=w_{1 c} z_{2 c}+z_{1 c} w_{2 c}^{*} e^{-i \kappa D}$, which (along with its complex conjugate) are the quantities in the parentheses in Eq. (10). Apparently, for the reflectionless state we have $z_{\text {tot }}=0$ and consequently Eq. (10) [and Eq. (15)] becomes zero. Therefore, also this $\kappa=k_{a}$ value will be unaffected by the $\ell$ variation and will appear as a horizontal line in the $\kappa$ vs $\ell$ diagram. Here, contrary to the SPTR, the distance $L$ plays a major role in the transparency, since an APTR corresponds to a specific distance $L$. This leads to fact that there is always a correspondence between an APTR of a scattering system at $k_{s}$ and a translation-invariant bound state of its bounded counterpart at $\kappa=k_{s}$.
Inversely, it holds that a translation-invariant bound state at $\kappa$ value will manifest in the transmittance of the corresponding scattering system as a PTR.

As a final comment, we stress that throughout our analysis we have employed transfer-matrix-based techniques, both for the numerical and for the analytical results. However, different and powerful techniques, such as Green's function methods $[38,42]$, could possibly be employed.

\section{CONCLUSION}

We have explored a generic bounded system with hardwall boundary conditions, consisting of two locally reflection symmetric potential barriers. Focusing on the variation of the energy eigenvalues by tuning the position of the potential units inside the box, we proved the existence of spectral extrema where the mirror symmetry of the wave function is restored inside each locally symmetric potential barrier. These extrema accumulate to eigenenergy values which coincide with the energies where perfect transmission resonances emerge in the transmittance of the associated scattering system. This behavior is a benchmark of the duality between scattering and bounded systems in the presence of locally symmetric potential landscapes. It is exemplified in this work for a system with two domains of local symmetry comprised of Dirac $\delta$ barriers. Our work could facilitate the design of cavities with prescribed spectral and wave-function properties. The established duality opens the perspective of linking and controlling bounded versus scattering setups. Not only can a bounded system be designed to possess LS symmetric wave functions, but its opening up to a scattering device leads also to an infinite-range extension via PTRs to the outside region.

\section{ACKNOWLEDGMENTS}

The authors thank V. Achilleos for fruitful discussions. This work was supported by the APAMAS project, which is supported by Le Mans Acoustique and funded by the Pays de la Loire region and the European Regional Development Fund.
[1] F. Bloch, Z. Phys. 52, 555 (1929).

[2] N. Zettili, Quantum Mechanics Concepts and Applications (Wiley, West Sussex, 2009).

[3] D. Shechtman, I. Blech, D. Gratias, and J. W. Cahn, Phys. Rev. Lett. 53, 1951 (1984).

[4] M. Widom, D. P. Deng, and C. L. Henley, Phys. Rev. Lett. 63, 310 (1989).

[5] B. Verberck, Nat. Phys. 10, 264 (2014).

[6] P. Wochner, C. Gutt, T. Autenrieth, T. Demmer, V. Bugaev, A. Díaz Ortiz, A. Duri, F. Zontone, G. Grübel, and H. Dosch, Proc. Natl. Acad. Sci. USA 106, 11511 (2009).

[7] K. Grzeskowiak, D. S. Goodsell, M. Kaczor-Grzeskowiak, D. Cascio, and R. E. Dickerson, Biochemistry 32, 8923 (1993).

[8] R. A. Pascal, J. Phys. Chem. A 105, 9040 (2001).

[9] P. Gipson, M. L. Baker, D. Raytcheva, C. Haase-Pettingell, J. Piret, J. A. King, and W. Chiu, Nat. Commun. 5, 4278 (2014).

[10] E. Macia, Rep. Prog. Phys. 69, 397 (2006).
[11] S. V. Zhukovsky, Phys. Rev. A 81, 053808 (2010).

[12] R. W. Peng, X. Q. Huang, F. Qiu, Mu Wang, A. Hu, S. S. Jiang, and M. Mazzer, Appl. Phys. Lett. 80, 3063 (2002).

[13] D. K. Ferry and S. M. Goodnick, Transport in Nanostructures (Cambridge University Press, Cambridge, 1997).

[14] W. J. Hsueh, C. H. Chen, and R. Z. Qiu, Phys. Lett. A 377, 1378 (2013).

[15] R. Hao, H. Jia, Y. Ye, F. Liu, C. Qiu, M. Ke, and Z. Liu, Europhys. Lett. 92, 24006 (2010).

[16] H. Aynaou, E. H. El Boudouti, B. Djafari-Rouhani, A. Akjouj, and V. R. Velasco, J. Phys.: Condens. Matter 17, 4245 (2005).

[17] P. D. C. King and T. J. Cox, J. Appl. Phys. 102, 014902 (2007).

[18] P. D. Hladky-Hennion, J. O. Vasseur, S. Degraeve, C. Granger, and M. de Billy, J. Appl. Phys. 113, 154901 (2013).

[19] S. Tamura and J. P. Wolfe, Phys. Rev. B 36, 3491 (1987).

[20] R. K. Mishra, K. D. Misra, and R. P. Tiwari, Surf. Rev. Lett. 11, 541 (2004). 
[21] P. A. Kalozoumis, C. Morfonios, F. K. Diakonos, and P. Schmelcher, Phys. Rev. Lett. 113, 050403 (2014).

[22] P. A. Kalozoumis, C. V. Morfonios, F. K. Diakonos, and P. Schmelcher, Ann. Phys. (NY) 362, 684 (2015).

[23] V. E. Zambetakis, M. K. Diakonou, P. A. Kalozoumis, F. K. Diakonos, C. V. Morfonios, and P. Schmelcher, J. Phys. A: Math. Theor. 49, 195304 (2016).

[24] C. Morfonios, P. Schmelcher, P. A. Kalozoumis, and F. K. Diakonos, Nonlinear Dyn. 78, 71 (2014).

[25] T. Wulf, C. V. Morfonios, F. K. Diakonos, and P. Schmelcher, Phys. Rev. E 93, 052215 (2016).

[26] P. A. Kalozoumis, C. Morfonios, F. K. Diakonos, and P. Schmelcher, Phys. Rev. A 87, 032113 (2013).

[27] P. A. Kalozoumis, C. Morfonios, N. Palaiodimopoulos, F. K. Diakonos, and P. Schmelcher, Phys. Rev. A 88, 033857 (2013).

[28] P. A. Kalozoumis, O. Richoux, F. K. Diakonos, G. Theocharis, and P. Schmelcher, Phys. Rev. B 92, 014303 (2015).

[29] C. V. Morfonios, P. A. Kalozoumis, F. K. Diakonos, and P. Schmelcher, Ann. Phys. (NY) 385, 623 (2017).

[30] M. Röntgen, C. V. Morfonios, and P. Schmelcher, Phys. Rev. B 97, 035161 (2018).
[31] M. Röntgen, C. V. Morfonios, R. Wang, L. Dal Negro, and P. Schmelcher, arXiv:1807.06812.

[32] C. V. Morfonios, M. Röntgen, F. K. Diakonos, and P. Schmelcher, arXiv:1807.02793.

[33] P. Ambichl, K. G. Makris, L. Ge, Y. Chong, A. D. Stone, and S. Rotter, Phys. Rev. X 3, 041030 (2013).

[34] H.-J. Stöckmann, E. Persson, Y.-H. Kim, M. Barth, U. Kuhl, and I. Rotter, Phys. Rev. E 65, 066211 (2002).

[35] E. Doron and U. Smilansky, Nonlinearity 5, 1055 (1992).

[36] B. Dietz and U. Smilansky, Chaos 3, 581 (1993).

[37] B. Dietz, J. P. Eckmann, C. A. Pillet, U. Smilansky, and I. Ussishkin, Phys. Rev. E 51, 4222 (1995).

[38] A. G. M. Schmidt, B. K. Cheng, and M. G. E. da Luz, Phys. Rev. A 66, 062712 (2002).

[39] M. G. E. Da Luz, E. J. Heller, and B. K. Cheng, J. Phys. A: Math. Gen. 31, 2975 (1998).

[40] X. Huang, Y. Wang, and C. Gong, J. Phys.: Condens. Matter 11, 7645 (1999).

[41] X. Q. Huang, S. S. Jiang, R. W. Peng, and A. Hu, Phys. Rev. B 63, 245104 (2001).

[42] F. M. Andrade, B. K. Cheng, M. W. Beims, and M. G. E. da Luz, J. Phys. A: Math. Gen. 36, 227 (2003). 